\title{
Dispersion and Absorption of Longitudinal Electro-Kinetic Waves in Magnetized Ion-Implanted Semiconductor Plasmas
}

\author{
S. Ghosh* And Preeti Thakur \\ School of Studies in Physics, Vikram University, Ujjain-456 010 (M.P.), India
}

(Received February 6, 2006)

\begin{abstract}
The instability of longitudinal electro-kinetic wave that is propagating across the magnetic field $\boldsymbol{B}_{0}$ in ion-implanted group-IV semiconductor sample consisting of non-drifting electrons, holes, and negatively charged colloids is investigated. Using hydrodynamic approach, a compact dispersion relation $D(\omega, \boldsymbol{k})$ is derived for the same. The propagation and amplification characteristics of the wave in collision dominated regime are studied numerically in detail for slow and fast modes. Results show that the presence of transverse magnetic field is responsible for the modification of the existing wave spectra of the slow electro-kinetic branch. In case of fast electro-kinetic wave, the presence of magnetic field is found responsible for excitation and amplification of a new mode. It is also found that the magnetic field is responsible for the conversion of two existing aperiodic modes into periodic ones. These novel periodic modes are found to be counter-propagating in nature.
\end{abstract}

PACS numbers: 52.35.Fp, 61.72.Ww, 82.70.Dd

\section{Introduction}

Very recently, the present authors have reported analytically the instability of new electro-kinetic [1,2], electro-acoustic waves [3, 4] and the modifications in existing wave spectra in colloids laden semiconductor plasma medium. The investigations in Refs. [1, 2] are found to be very interesting and useful in understanding the characteristics of longitudinal electro-kinetic waves (LEKW) and the novel properties introduced due to the presence of negatively charged colloids in unmagnetized semiconductor plasma consisting of electrons, holes, and colloids.

*corresponding author; e-mail: drsanjayghosh@rediffmail.com 
The most important distinguishable feature of this medium from other usual multi-component plasma media, are the presence of comparatively massive charged colloids which introduce new time and space scales in plasma behavior leading to novel wave modes in addition to modifications of existing wave spectra and instability phenomena [1-6]. This medium is now becoming friendlier to technologists because of its immense applications in processing of new materials and fabrication of improved devices [7-9].

The current investigations in this direction indicate that the presence of charged colloids has a strong influence on the characteristics of usual plasma wave modes even at frequency where colloidal grains do not participate in wave motion.

With the solid as a plasma source, the properties of electrons, holes and interacting excitations are well known and controllable. The major practical interest in the field of wave instabilities is the possibility of adjusting the field geometries and physical conditions to maximize wave gain and thus derive from the solid state plasmas useful intensities of power in favorable frequency regime. It is a well-known fact that the application of magnetic field adds new dimensions to the field of waves and instabilities in solid-state plasma.

In the light of the above, we intend to report the investigations made on the dispersion and absorption characteristics of the fundamental LEKW in magnetized semiconductor plasma medium, laden with negatively charged colloids, those are assumed to be stationary forming neutralizing background in the medium. Finally, we have done an exhaustive numerical analysis with a set of data appropriate for a group-IV semiconductor crystal to establish the validity of analysis and provided brief conclusions for the results obtained.

\section{Theoretical formulation}

This section is devoted to theoretical formulation, in which we have considered the well-known hydrodynamical model of a homogeneous multi-component semiconductor plasma of infinite extent, consisting of non-drifting electrons, holes, and stationary but participating negatively charged colloids. The medium is considered to be a group-IV semiconductor sample immersed in static magnetic field $\boldsymbol{B}_{0}$ pointing perpendicular to the propagation direction ( $x$-axis). Here the use of hydrodynamic model allows us to replace the carrier plasma by a charged fluid characterized by a few macroscopic parameters like mean carrier density, mean velocity, etc. of the plasma fluid and this makes the analysis of LEKW interaction and other related phenomena simple.

The colloids that act as third species or foreign particles are the result of the implantation of any metal ion (such as $\mathrm{Ag}^{+}, \mathrm{Cu}^{+}, \mathrm{Fe}^{+}$, etc.) inside the medium [10-12]. These colloids acquire a negative charge through the sticking of high mobility free electrons on them. These negatively charged colloids are assumed to be of uniform size and smaller than both the wavelength under study and the carrier Debye radii; hence can be treated as negatively charge point masses. These colloidal particles have a significant effect on the behavior of plasma. 
Presently, we shall study the linear dispersion relation for low frequency electrostatic wave propagating perpendicular to $\boldsymbol{B}_{0}$. In the equilibrium, the plasma is quasi-neutral and the conservation of particle number density must always hold. Thus

$$
n_{0 \mathrm{~h}}=n_{0 \mathrm{e}}+z_{\mathrm{d}} n_{0 \mathrm{~d}},
$$

where $z_{\mathrm{d}}\left(=q_{\mathrm{d}} / e\right)$ refers to charge state of colloids, i.e. the ratio of negative charges $q_{\mathrm{d}}$ residing on the colloidal grains to the charge $e$ of the electrons; $n_{0 \mathrm{e}, \mathrm{h}, \mathrm{d}}$ are unperturbed particle number densities of electrons, holes, and colloids, respectively.

The basic equations governing the LEKW instability in colloids laden magnetized semiconductor plasma for electrons and holes, respectively, are: momentum transfer equation

$$
\begin{gathered}
\frac{\partial \boldsymbol{\vartheta}_{1 \mathrm{e}}}{\partial t}+\nu_{\mathrm{e}} \boldsymbol{\vartheta}_{1 \mathrm{e}}=\frac{-e}{m_{\mathrm{e}}}\left(\boldsymbol{E}_{1}+\boldsymbol{\vartheta}_{1 \mathrm{e}} \times \boldsymbol{B}_{0}\right)-\frac{\vartheta_{l \mathrm{e}}^{2}}{\rho_{0 \mathrm{e}}} \frac{\partial \rho_{1 \mathrm{e}}}{\partial x} \\
\frac{\partial \boldsymbol{\vartheta}_{1 \mathrm{~h}}}{\partial t}+\nu_{\mathrm{h}} \boldsymbol{\vartheta}_{1 \mathrm{~h}}=\frac{e}{m_{\mathrm{h}}}\left(\boldsymbol{E}_{1}+\boldsymbol{\vartheta}_{1 \mathrm{~h}} \times \boldsymbol{B}_{0}\right)-\frac{\vartheta_{l \mathrm{~h}}^{2}}{\rho_{0 \mathrm{~h}}} \frac{\partial \rho_{1 \mathrm{~h}}}{\partial x},
\end{gathered}
$$

continuity equation

$$
\begin{aligned}
& \frac{\partial n_{1 \mathrm{e}}}{\partial t}+n_{0 \mathrm{e}} \nabla \boldsymbol{\vartheta}_{1 \mathrm{e}}=0, \\
& \frac{\partial n_{1 \mathrm{~h}}}{\partial t}+n_{0 \mathrm{~h}} \nabla \boldsymbol{\vartheta}_{1 \mathrm{~h}}=0,
\end{aligned}
$$

wave equation

$$
\boldsymbol{k} \times \boldsymbol{k} \times \boldsymbol{E}_{1}=\mathrm{i} \omega \mu_{0} \boldsymbol{J}_{1}-\frac{\omega^{2}}{c_{\mathrm{L}}^{2}} \boldsymbol{E}_{1}=0 .
$$

where $m_{\mathrm{e}, \mathrm{h}}, \boldsymbol{\vartheta}_{1 \mathrm{e}, \mathrm{h}}, \nu_{\mathrm{e}, \mathrm{h}}$, and $\vartheta_{\text {te,h }}$ are the masses, velocities, phenomenological collision frequencies, and thermal velocities of electrons and holes, respectively. All the other symbols have their usual meanings. Here the suffixes 0 and 1 used in above equations indicate a steady state (zeroth order) and time varying (first order) quantities, respectively.

Assuming the first order quantities varying as $\exp [\mathrm{i}(\omega t-k x)]$ (where $\omega$ and $k$ are frequency and wave number of the propagating mode, respectively), and following the procedure adopted by Chen [10] and Steele and Vural [11], the modified dielectric response function of LEKW in colloids laden magnetized semiconductor plasma medium is obtained as

$$
\begin{aligned}
& \varepsilon(\omega, \boldsymbol{k})=1+\frac{\omega_{\mathrm{pe}}^{2}\left(\omega-\mathrm{i} \nu_{\mathrm{e}}\right)}{\left(\omega-\mathrm{i} \nu_{\mathrm{e}}\right)\left(\omega^{2}-\mathrm{i} \omega \nu_{\mathrm{e}}-k^{2} \vartheta_{\mathrm{te}}^{2}\right)-\omega \omega_{\mathrm{ce}}^{2}} \\
& \quad+\frac{\omega_{\mathrm{ph}}^{2}\left(\omega-\mathrm{i} \nu_{\mathrm{h}}\right)}{\left(\omega-\mathrm{i} \nu_{\mathrm{h}}\right)\left(\omega^{2}-\mathrm{i} \omega \nu_{\mathrm{h}}-k^{2} \vartheta_{\mathrm{th}}^{2}\right)-\omega \omega_{\mathrm{ch}}^{2}}+\frac{\omega_{\mathrm{pd}}^{2}}{\left(\omega^{2}-\omega_{\mathrm{cd}}^{2}\right)}=0
\end{aligned}
$$

where $\omega_{\mathrm{pe}, \mathrm{h}}^{2}=e^{2} n_{0 \mathrm{e}, \mathrm{h}} / \varepsilon m_{\mathrm{e}, \mathrm{h}}, \quad \omega_{\mathrm{pd}}^{2}=\left(z_{\mathrm{d}} e\right)^{2} n_{0 \mathrm{~d}} / \varepsilon m_{\mathrm{d}}, \quad \omega_{\mathrm{ce}, \mathrm{h}}=e B_{0} / m_{\mathrm{e}, \mathrm{h}}$, $\omega_{\mathrm{cd}}=z_{\mathrm{d}} e B_{0} / m_{\mathrm{d}}, \vartheta_{\mathrm{te}, \mathrm{h}}^{2}=k_{\mathrm{B}} T / m_{\mathrm{e}, \mathrm{h}}$ and $\varepsilon=\varepsilon_{0} \varepsilon_{\mathrm{L}}, \varepsilon_{\mathrm{L}}$ being the lattice dielectric constant. 
If one neglects the carrier cyclotron frequency $\omega_{\text {ce,h,d }}\left(B_{0}=0\right)$, the above equation reduces to Eq. (4) of Ref. [1]. Now we concentrate towards the principle aim of this paper, i.e. instability characteristics of electro-kinetic (EK) mode in ion-implanted semiconductor plasma in the presence of external magnetic field $\boldsymbol{B}_{0}$ applied perpendicularly to the wave propagation direction.

We consider two interesting cases of important parameter regimes, which can be satisfied easily in the present host medium.

Case 1: slow electro-kinetic mode $\left(\omega \ll k \vartheta_{\mathrm{te}}, k \vartheta_{\mathrm{th}}\right)$

Under collision dominated or low frequency regime $\left(\omega \ll \nu_{\mathrm{e}}, \nu_{\mathrm{h}}\right)$, if the phase velocity of the wave is smaller than the thermal velocities of both electrons and holes, then the mode may be termed as slow electro-kinetic mode (SEKM). For this case the dispersion relation (7) reduces to

$$
\begin{aligned}
& 1-\frac{\mathrm{i} \nu_{\mathrm{e}} \omega_{\mathrm{pe}}^{2}}{\omega\left(\nu_{\mathrm{e}}^{2}+\omega_{\mathrm{ce}}^{2}\right)-\mathrm{i} k^{2} \lambda_{\mathrm{De}}^{2} \omega_{\mathrm{pe}}^{2} \nu_{\mathrm{e}}}-\frac{\mathrm{i} \nu_{\mathrm{h}} \omega_{\mathrm{ph}}^{2}}{\omega\left(\nu_{\mathrm{h}}^{2}+\omega_{\mathrm{ch}}^{2}\right)-\mathrm{i} k^{2} \lambda_{\mathrm{Dh}}^{2} \omega_{\mathrm{ph}}^{2} \nu_{\mathrm{h}}}+ \\
& \quad+\frac{\omega_{\mathrm{pe}}^{2}}{\omega^{2}-\omega_{\mathrm{cd}}^{2}}=0
\end{aligned}
$$

where $\lambda_{\text {De,h }}^{2}=\vartheta_{\text {te,h }} / \omega_{\text {pe,h }}$, square of the Debye lengths for electrons and holes.

Equation (8) may be written in the form of polynomial in $\omega$ as

$$
\begin{aligned}
& \omega^{4}\left(\nu_{\mathrm{e}}^{2}+\omega_{\mathrm{ce}}^{2}\right)\left(\nu_{\mathrm{h}}^{2}+\omega_{\mathrm{ch}}^{2}\right) \\
& \quad-\mathrm{i} \omega^{3}\left[\left(\nu_{\mathrm{e}}^{2}+\omega_{\mathrm{ce}}^{2}\right)\left(k^{2} \lambda_{\mathrm{Dh}}^{2}-1\right) \omega_{\mathrm{ph}}^{2} \nu_{\mathrm{h}}+\left(\nu_{\mathrm{h}}^{2}+\omega_{\mathrm{ch}}^{2}\right)\left(k^{2} \lambda_{\mathrm{De}}^{2}-1\right) \omega_{\mathrm{pe}}^{2} \nu_{\mathrm{e}}\right] \\
& +\omega^{2}\left[\left(\nu_{\mathrm{e}}^{2}+\omega_{\mathrm{ce}}^{2}\right)\left(\nu_{\mathrm{h}}^{2}+\omega_{\mathrm{ch}}^{2}\right)\left(\omega_{\mathrm{pd}}^{2}-\omega_{\mathrm{cd}}^{2}\right)\right. \\
& \left.+k^{2}\left(\lambda_{\mathrm{De}}^{2}+\lambda_{\mathrm{Dh}}^{2}-k^{2} \lambda_{\mathrm{De}}^{2} \lambda_{\mathrm{Dh}}^{2}\right) \omega_{\mathrm{pe}}^{2} \omega_{\mathrm{ph}}^{2} \nu_{\mathrm{e}} \nu_{\mathrm{h}}\right] \\
& +\mathrm{i} \omega\left\{\omega_{\mathrm{ph}}^{2} \nu_{\mathrm{h}}\left(\nu_{\mathrm{e}}^{2}+\omega_{\mathrm{ce}}^{2}\right)\left[\left(k^{2} \lambda_{\mathrm{Dh}}^{2}-1\right) \omega_{\mathrm{cd}}^{2}-k^{2} \lambda_{\mathrm{Dh}}^{2} \omega_{\mathrm{pd}}^{2}\right]\right. \\
& \left.+\omega_{\mathrm{pe}}^{2} \nu_{\mathrm{e}}\left(\nu_{\mathrm{h}}^{2}+\omega_{\mathrm{ch}}^{2}\right)\left[\left(k^{2} \lambda_{\mathrm{De}}^{2}-1\right) \omega_{\mathrm{cd}}^{2}-k^{2} \lambda_{\mathrm{De}}^{2} \omega_{\mathrm{pd}}^{2}\right]\right\} \\
& +k^{2}\left[k^{2} \lambda_{\mathrm{De}}^{2} \lambda_{\mathrm{Dh}}^{2} \omega_{\mathrm{pe}}^{2} \omega_{\mathrm{ph}}^{2} \nu_{\mathrm{e}} \nu_{\mathrm{h}}\left(\omega_{\mathrm{cd}}^{2}-\omega_{\mathrm{pd}}^{2}\right)\right. \\
& \left.\quad-\omega_{\mathrm{pe}}^{2} \omega_{\mathrm{ph}}^{2} \nu_{\mathrm{e}} \nu_{\mathrm{h}} \omega_{\mathrm{cd}}^{2}\left(\lambda_{\mathrm{De}}^{2}+\lambda_{\mathrm{Dh}}^{2}\right)\right]=0 .
\end{aligned}
$$

One can infer that in the absence of applied magnetic field $\left(\omega_{\text {ce,h,d }}=0\right)$, the polynomial (9) reduces to Eq. (6a) of Ref. [1]. Hence it is very clearly demonstrated in Eq. (9) that the presence of externally applied magnetic field effectively modifies the wave spectra of all the four possible existing modes.

$$
\text { Case 2: fast electro-kinetic mode }\left(k \vartheta_{\text {th }} \ll \omega \ll k \vartheta_{\text {te }}\right)
$$

Under collision dominated or low frequency regime $\left(\omega \ll \nu_{\mathrm{e}}, \nu_{\mathrm{h}}\right)$, if the phase velocity of the wave is smaller than the thermal velocities of electrons but more than the hole thermal velocity, the mode may be termed as fast electro-kinetic 
mode (FEKM). For FEKM the dispersion relation (7) reduces to

$$
1-\frac{\mathrm{i} \nu_{\mathrm{e}} \omega_{\mathrm{pe}}^{2}}{\omega\left(\nu_{\mathrm{e}}^{2}+\omega_{\mathrm{ce}}^{2}\right)-\mathrm{i} k^{2} \lambda_{\mathrm{De}}^{2} \omega_{\mathrm{pe}}^{2} \nu_{\mathrm{e}}}-\frac{\mathrm{i} \nu_{\mathrm{h}} \omega_{\mathrm{ph}}^{2}}{\omega\left(\nu_{\mathrm{h}}^{2}+\omega_{\mathrm{ch}}^{2}\right)+\mathrm{i} \omega^{2} \nu_{\mathrm{h}}}+\frac{\omega_{\mathrm{pd}}^{2}}{\omega^{2}-\omega_{\mathrm{cd}}^{2}}=0 .
$$

In the form of polynomial in $\omega$ Eq. (10) may be written as

$$
\begin{aligned}
\mathrm{i} \omega^{5}[ & \left.\nu_{\mathrm{h}}\left(\nu_{\mathrm{e}}^{2}+\omega_{\mathrm{ce}}^{2}\right)\right]+\omega^{4}\left[\left(\nu_{\mathrm{e}}^{2}+\omega_{\mathrm{ce}}^{2}\right)\left(\nu_{\mathrm{h}}^{2}+\omega_{\mathrm{ch}}^{2}\right)+\left(k^{2} \lambda_{\mathrm{De}}^{2}-1\right) \omega_{\mathrm{pe}}^{2} \nu_{\mathrm{e}} \nu_{\mathrm{h}}\right] \\
& \left.-\mathrm{i} \omega^{3}\left(k^{2} \lambda_{\mathrm{De}}^{2}-1\right)\left(\nu_{\mathrm{h}}^{2}+\omega_{\mathrm{ch}}^{2}\right) \omega_{\mathrm{pe}}^{2} \nu_{\mathrm{e}}+\nu_{\mathrm{h}}\left(\nu_{\mathrm{e}}^{2}+\omega_{\mathrm{ce}}^{2}\right)\left(\omega_{\mathrm{cd}}^{2}-\omega_{\mathrm{ph}}^{2}-\omega_{\mathrm{pd}}^{2}\right)\right] \\
& -\omega^{2}\left[\left(\nu_{\mathrm{e}}^{2}+\omega_{\mathrm{ce}}^{2}\right)\left(\nu_{\mathrm{h}}^{2}+\omega_{\mathrm{ch}}^{2}\right)\left(\omega_{\mathrm{cd}}^{2}-\omega_{\mathrm{pd}}^{2}\right)\right. \\
& \left.+k^{2} \lambda_{\mathrm{De}}^{2} \omega_{\mathrm{pe}}^{2} \nu_{\mathrm{e}} \nu_{\mathrm{h}}\left(\omega_{\mathrm{cd}}^{2}-\omega_{\mathrm{ph}}^{2}-\omega_{\mathrm{pd}}^{2}\right)-\omega_{\mathrm{pe}}^{2} \omega_{\mathrm{cd}}^{2} \nu_{\mathrm{e}} \nu_{\mathrm{h}}\right] \\
& +\mathrm{i} \omega\left[\left(\nu_{\mathrm{h}}^{2}+\omega_{\mathrm{ch}}^{2}\right)\left(\omega_{\mathrm{cd}}^{2}-\omega_{\mathrm{pd}}^{2}\right) k^{2} \lambda_{\mathrm{De}}^{2} \omega_{\mathrm{pe}}^{2} \nu_{\mathrm{e}}-\omega_{\mathrm{pe}}^{2} \omega_{\mathrm{cd}}^{2} \nu_{\mathrm{e}}\left(\nu_{\mathrm{h}}^{2}+\omega_{\mathrm{ch}}^{2}\right)\right. \\
& \left.-\omega_{\mathrm{ph}}^{2} \omega_{\mathrm{cd}}^{2} \nu_{\mathrm{h}}\left(\nu_{\mathrm{e}}^{2}+\omega_{\mathrm{ce}}^{2}\right)\right]-k^{2} \lambda_{\mathrm{De}}^{2} \omega_{\mathrm{pe}}^{2} \omega_{\mathrm{ph}}^{2} \nu_{\mathrm{e}} \nu_{\mathrm{h}} \omega_{\mathrm{cd}}^{2}=0 .
\end{aligned}
$$

In the absence of applied magnetic field $\left(\omega_{\mathrm{ce}, \mathrm{h}, \mathrm{d}}=0\right)$, the above equation reduces to Eq. (8a) of Ref. [1]. It can be inferred, comparing Eq. (11) with Eq. (8a) of Ref. [1], that the presence of magnetic field $\boldsymbol{B}_{0}$ does not only introduce one new branch of propagation for FEKW in the host medium but also modifies the spectra of already available modes. To study the temporal instability and dispersion characteristics Eqs. (9) and (11) being fourth and fifth order polynomials can be solved numerically by considering $k$ as real positive quantity and $\omega$ complex.

\section{Result and discussions}

To have numerical appreciations of the dispersion relations so obtained in the theoretical formulation, we have used the physical parameters suitable for Ge crystal as: $m_{\mathrm{e}}=0.0815 m_{0}, m_{0}$ being the free electron mass, $m_{\mathrm{h}}=4 m_{\mathrm{e}}$, $m_{\mathrm{d}}=10^{-27} \mathrm{~kg}, \varepsilon_{\mathrm{L}}=15.8, n_{0 \mathrm{e}}=10^{19} \mathrm{~m}^{-3}, n_{0 \mathrm{~h}}=5 \times 10^{19} \mathrm{~m}^{-3}, n_{0 \mathrm{~d}}=10^{14} \mathrm{~m}^{-3}$, $z_{\mathrm{d}}=10^{2}, \nu_{\mathrm{e}}=3.463 \times 10^{11} \mathrm{~s}^{-1}, \nu_{\mathrm{h}}=1.194 \times 10^{11} \mathrm{~s}^{-1}$ and $\nu_{\mathrm{d}}=3.422 \times 10^{8} \mathrm{~s}^{-1}$. We have used Eqs. (9) and (11) for the numerical calculations and the results are displayed in the form of curves in Figs. 1-3 for SEKM and in the form of Tables I and II for FEKM.

The first order perturbation is assumed to vary as $\exp [\mathrm{i}(\omega t-k x)]$; so the wave may be growing in time when the imaginary part of the wave frequency $\omega_{\mathrm{i}}<0$ (i.e. the wave is extracting power from the medium) which causes a temporal instability. On the other hand, the wave may be decaying in time when $\omega_{\mathrm{i}}>0$ (i.e. the power is being absorbed by the wave from the medium) so that the wave may suffer an attenuation.

The variations of growth rate $\left(\omega_{\mathrm{i}}\right)$ with wave number $(k)$ for the first and second aperiodic modes for SEKM are displayed in Fig. 1. These modes remain aperiodic in nature in the presence as well as in the absence of magnetic field. 


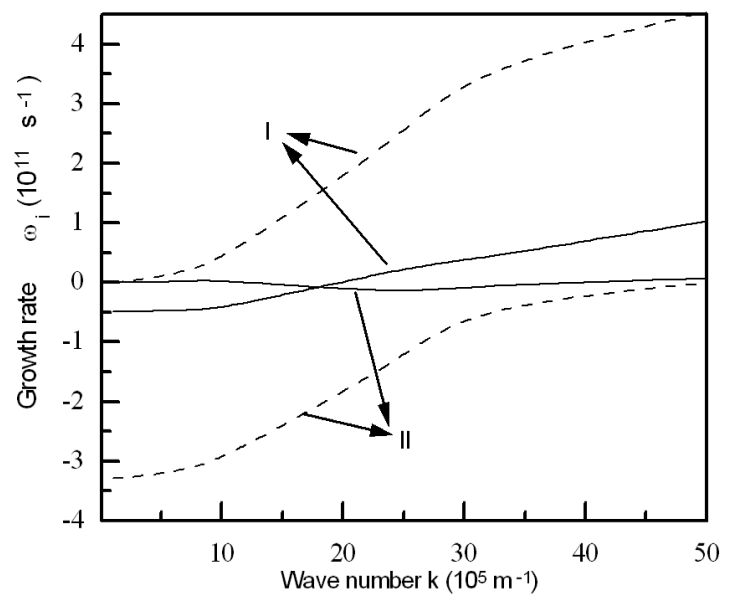

Fig. 1. Variation of growth rate of first and second modes with wave number $k ;(-)$ $B_{0}=0.5 \mathrm{~T},(-\cdots) B_{0}=0$.

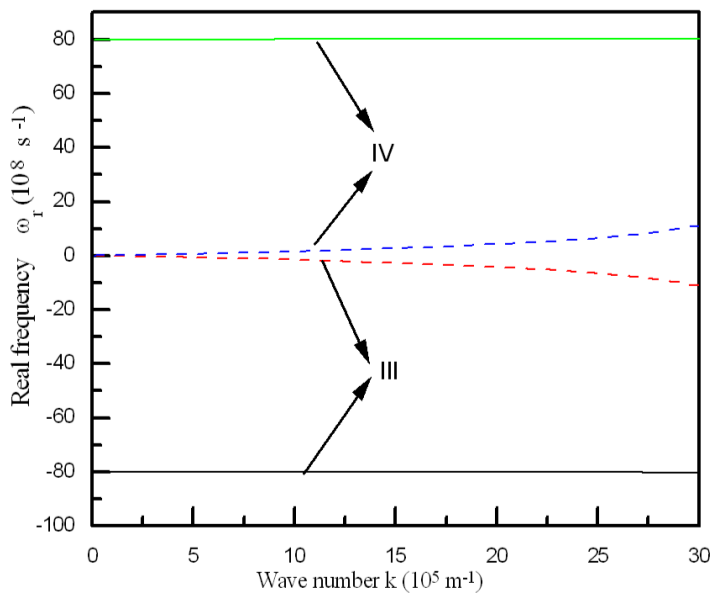

Fig. 2. Variation of real frequency of third and fourth periodic modes with wave number $k ;(-) B_{0}=0.5 \mathrm{~T},(----) B_{0}=0$.

Thus it can be said that with the application of magnetic field, the dispersion characteristics of these two modes do not change their nature.

Figure 1 illustrates that in the absence of externally applied magnetic field $\left(B_{0}=0\right)$, the first/second mode shows attenuation/amplification characteristic with decay/growth rate increases/decreases with increasing the value of wave number. In the presence of magnetic field $\left(B_{0}=0.5 \mathrm{~T}\right)$, the first mode changes its characteristic to amplification up to $k \leq 1.76 \times 10^{6} \mathrm{~m}^{-1}$ and beyond this point it starts decaying. Even though the decay coefficient approaches a negligible value, the nature of the second mode does not change in the presence of $\boldsymbol{B}_{0}$. Hence the 


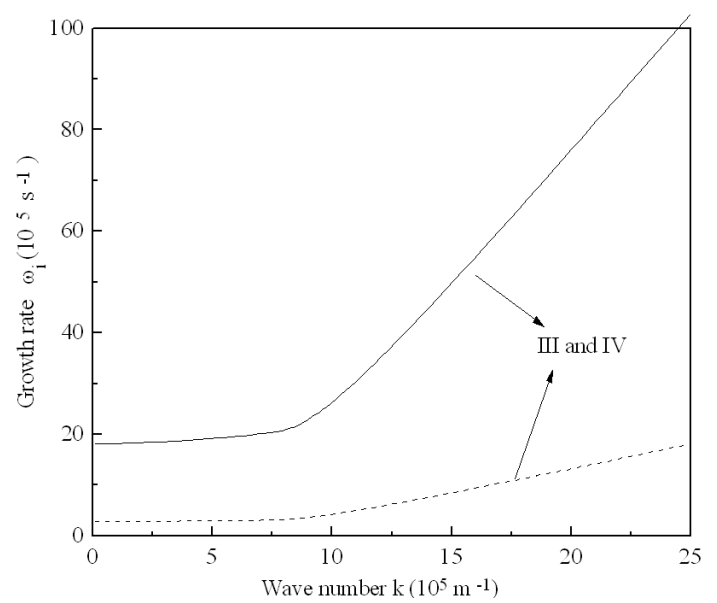

Fig. 3. Variation of growth rate of third and fourth modes with wave number $k ;(-)$ $B_{0}=0.5 \mathrm{~T},(----) B_{0}=0$.

TABLE I

Variation of real part of the wave frequency $\left(\omega_{\mathrm{r}}\right)$ with $k$ for fast electro-kinetic wave. The I and II aperiodic modes $\left(\omega_{\mathrm{r}}=0\right)$ remain aperiodic even in the presence of magnetic field.

\begin{tabular}{c|c|c|c|c|c}
\hline \hline \multirow{2}{*}{$\begin{array}{c}\text { Wave number } \\
k\left[10^{4} \mathrm{~m}^{-1}\right]\end{array}$} & \multicolumn{5}{|c}{ Real part of wave frequency $\omega_{\mathrm{r}}\left[\mathrm{s}^{-1}\right]$} \\
\cline { 2 - 6 } & \multicolumn{2}{|c|}{ III-root } & \multicolumn{2}{|c}{ IV-root } & V-root \\
\cline { 2 - 6 } & $B_{0}=0$ & $B_{0}=0.5 \mathrm{~T}$ & $B_{0}=0$ & $B_{0}=0.5 \mathrm{~T}$ & $B_{0}=0.5 \mathrm{~T}$ \\
\hline 1 & 0 & $-8 \times 10^{9}$ & 0 & $8 \times 10^{9}$ & 30.439 \\
2.5 & 0 & $-8 \times 10^{9}$ & 0 & $8 \times 10^{9}$ & 30.44 \\
5 & 0 & $-8 \times 10^{9}$ & 0 & $8 \times 10^{9}$ & 30.445 \\
7.5 & 0 & $-8 \times 10^{9}$ & 0 & $8 \times 10^{9}$ & 30.453 \\
10 & 0 & $-8 \times 10^{9}$ & 0 & $8 \times 10^{9}$ & 30.464 \\
25 & 0 & $-8 \times 10^{9}$ & 0 & $8 \times 10^{9}$ & 30.594 \\
50 & 0 & $-8 \times 10^{9}$ & 0 & $8 \times 10^{9}$ & 31.057 \\
75 & 0 & $-8 \times 10^{9}$ & 0 & $8 \times 10^{9}$ & 31.817 \\
100 & 0 & $-8 \times 10^{9}$ & 0 & $8 \times 10^{9}$ & 32.859
\end{tabular}

magnetic field is responsible for effective modification in the amplification characteristics of these two aperiodic modes.

Figures 2 and 3 display the variations of real and imaginary parts of wave frequency $\omega$, of third and fourth modes of SEKM, respectively, with $k$ using $B_{0}$ as a parameter. Figure 2 illustrates that both the modes have exactly opposite nature of variations of $\omega_{\mathrm{r}}$ with $k$ in the presence as well as in the absence of magnetic field, i.e. if one mode propagates along positive $x$-direction then other one propagates along negative $x$-direction. But both have identical magnitudes 
TABLE II

Variation of imaginary part of the wave frequency $\left(\omega_{\mathrm{i}}\right)$ with $k$ for fast electro-kinetic wave.

\begin{tabular}{c|c|c|c|c|c|c}
\hline \multirow{2}{*}{$\begin{array}{c}\text { Wave } \\
\text { number }\end{array}$} & \multicolumn{5}{|c}{ Imaginary part of wave frequency $\omega_{\mathrm{i}}\left[\mathrm{s}^{-1}\right]$} \\
\cline { 2 - 7 }$k\left[10^{4} \mathrm{~m}^{-1}\right]$ & \multicolumn{2}{|c|}{ I-root } & \multicolumn{2}{|c}{ II-root } & \multicolumn{2}{c}{ III-root } \\
\cline { 2 - 7 } & $B_{0}=0$ & $B_{0}=0.5 \mathrm{~T}$ & $B_{0}=0$ & $B_{0}=0.5 \mathrm{~T}$ & $B_{0}=0$ & $B_{0}=0.5 \mathrm{~T}$ \\
\cline { 2 - 7 } & $\times 10^{11}$ & $\times 10^{5}$ & $\times 10^{6}$ & $\times 10^{11}$ & $\times 10^{11}$ & $\times 10^{11}$ \\
\hline 1 & -1.756 & 3.345 & 3.041 & 7.66 & 2.238 & 1.812 \\
2.5 & -1.756 & 20.9 & 2.01 & 7.66 & 2.238 & 1.812 \\
5 & -1.756 & 83.62 & 80.87 & 7.66 & 2.238 & 1.812 \\
7.5 & -1.756 & 188.1 & 182.2 & 7.66 & 2.238 & 1.812 \\
10 & -1.756 & 334.5 & 324.2 & 7.66 & 2.238 & 1.815 \\
25 & -1.748 & 2092 & 2036 & 7.66 & 2.229 & 1.825 \\
50 & -1.726 & 8385 & 8273 & 7.66 & 2.217 & 1.848 \\
75 & -1.693 & 18930 & 19090 & 7.66 & 2.196 & 1.887 \\
100 & -1.652 & 33800 & 35110 & 7.66 & 2.247 & 2.079
\end{tabular}

TABLE II continued

Variation of imaginary part of the wave frequency $\left(\omega_{\mathrm{i}}\right)$ with $k$ for fast electro-kinetic wave.

\begin{tabular}{c|c|c|c}
\hline \hline \multirow{2}{*}{$\begin{array}{c}\text { Wave } \\
\text { number } \\
k\left[10^{4} \mathrm{~m}^{-1}\right]\end{array}$} & \multicolumn{3}{|c}{ Imaginary part of wave frequency... } \\
\cline { 2 - 4 } & \multicolumn{2}{|c}{ IV-root } & V-root \\
\cline { 2 - 4 } & $B_{0}=0$ & $B_{0}=0.5 \mathrm{~T}$ & $B_{0}=0.5 \mathrm{~T}$ \\
\cline { 2 - 4 }$\times 10^{5}$ & $\times 10^{11}$ & $\times 10^{10}$ \\
\hline 1 & 7.558 & 1.812 & -4.649 \\
2.5 & 7.148 & 1.812 & -4.649 \\
5 & 7.106 & 1.812 & -4.649 \\
7.5 & 7.098 & 1.812 & -4.649 \\
10 & 7.095 & 1.815 & -4.648 \\
25 & 7.093 & 1.825 & -4.646 \\
50 & 7.092 & 1.848 & -4.636 \\
75 & 7.092 & 1.887 & -4.62 \\
100 & 7.092 & 2.079 & -4.6
\end{tabular}

of phase velocities. It may also be seen from this graph that the presence of $B_{0}$ increases the magnitude of phase constants of both the modes but the nature of variation does not change. Hence, in the presence of magnetic field these two counter-propagating modes move faster than before in their respective directions.

From Fig. 3, one may infer that both the modes (third and fourth) show decaying nature, with an identical magnitude of gain coefficient in the presence 
as well as in the absence of magnetic field. The gain coefficients in the presence of magnetic field increase slowly up to $k \approx 10^{6} \mathrm{~m}^{-1}$ and for $k>10^{6} \mathrm{~m}^{-1}$ they start increasing sharply. Hence the presence of transverse magnetic field in colloids laden semiconductor plasma is responsible for enhancement of phase speeds as well as magnitude of attenuation coefficients of third and fourth SEKW.

The roots of dispersion relation of FEKW (Eq. (11)) in magnetized ion-implanted semiconductor plasma have been numerically presented in Tables I and II. Table I shows the variations of phase constants of all possible modes with wave number $k$, for $B_{0}=0$ and $B_{0}=0.5 \mathrm{~T}$. It is found that the existing four modes are aperiodic $\left(\omega_{\mathrm{r}}=0\right)$ in nature in the absence of magnetic field, whereas in the presence of $B_{0}$ the third and fourth modes are converted to periodic modes with an opposite and equal value of phase velocity. Their phase coefficients also remain unaffected with wave number. On the applications of $B_{0}$, a new (fifth) mode comes into existence, which is found to be periodic in nature with phase velocity marginally increasing in nature with $k$.

Table II describes the variation of growth rates of all possible modes with $k$ for $B_{0}=0$ and $B_{0}=0.5 \mathrm{~T}$. In the absence of magnetic field, out of the four existing modes only one (first) shows amplification characteristics whereas in the presence of magnetic field all of these four modes show decaying characteristics. The novel mode (fifth) introduced due to the presence of $B_{0}$ shows amplification characteristics whose gain coefficient is nearly unaffected by the wave number regime under study.

\section{Conclusions}

We have investigated the effect of transverse magnetic field on the excitation and amplification of LEKW in uniform group-IV semiconductor plasma consisting of participating negatively charged colloids. We focused over attention in two different regimes in which phase velocity of the wave is either smaller or larger than the thermal velocity of hole. The most important inferences that may be drawn from the above study are listed below.

1. For SEK branch, the presence of magnetic field is responsible for effective modification in the amplification characteristics of first two aperiodic modes and enhances the phase speeds of third and fourth modes.

2. For FEK branch, the presence of $B_{0}$ introduces a new mode into the medium. This new mode is found to be the only growing mode in the presence of $B_{0}$, which is propagating towards positive $x$-direction.

3. The magnetic field is responsible for converting third and fourth aperiodic modes of FEKW into periodic and counter-propagating modes having equal phase speed.

Thus the present theory provides a qualitative picture of wave spectrum of LEKW in magnetized, colloids laden semiconductor plasma. Authors not only 
found important modifications in EKW spectrum but also the existence of a new mode is established. Thus, for the experimental verification of our theoretical idea, we propose to initiate a serious laboratory experimental effort.

\section{Acknowledgments}

One of the authors (P.T.) is indebted to Ms. Pragati Khare for many stimulating discussions during the course of this work.

\section{References}

[1] S. Ghosh, Preeti Thakur, Eur. Phys. J. D 31, 85 (2004).

[2] S. Ghosh, Preeti Thakur, Eur. Phys. J. D 35, 449 (2005).

[3] S. Ghosh, G.R. Sharma, Pragati Khare, M. Salimullah, Physica B 351, 163 (2004).

[4] S. Ghosh, Pragati Khare, Eur. Phys. J. D 35, 521 (2005).

[5] S. Ghosh, Preeti Thakur, Eur. Phys. J. D 37, 417 (2006).

[6] S. Ghosh, Pragati Khare, Acta Phys. Pol. A 109, 187 (2006).

[7] C.W. White, J.D. Budal, J.G. Zhen, S.P. Withrow, Appl. Phys. Lett. 68, 2389 (1998).

[8] R.F. Huglum Jr., L. Yang, R.H. Magrunder III, J.E. Witting, K. Becker, R.A. Zuhr, Opt. Lett. 18, 373 (1993).

[9] S.M. Sze, Semiconductor Devices: Physics and Technology, J. Wiley, India 2002.

[10] F.F. Chen, Introduction to Plasma Physics and Controlled Fusion, Vol. 1, Plenum Press, New York 1984, p. 100.

[11] M.C. Steele, B. Vural, Wave Interactions in Solid State Plasmas, Mc-Graw Hill, New York 1969, p. 45.

[12] G.L. Zhang, W.H. Liu, F. Xu, W.X. Hu, Appl. Phys. Lett. 61, 2527 (1992).

[13] H. Hosono, Y. Abe, N. Matsunami, Appl. Phys. Lett. 60, 2613 (1992).

[14] N. Matsunami, H. Hosono, Appl. Phys. Lett. 63, 2050 (1993). 